\title{
Pobreza rural multidimensional en Zacatecas
}

\author{
MIREYA TORRES RAMÍREZ* \\ JUAN MANUEL PADILLA**
}

RESUMEN: Este trabajo presenta los resultados de la medición de la pobreza rural en el estado de Zacatecas, con base en una encuesta diseñada para tal fin. Los datos se refieren a 2009 y se usa el enfoque multidimensional propuesto por el Coneval. El trabajo se conforma por tres apartados: en el primero se discute el concepto de pobreza; en el segundo se presentan datos relevantes sobre ésta y de población a nivel nacional y para Zacatecas; en el tercero se analizan los resultados de las mediciones de pobreza rural en la entidad. El trabajo se centra en la discusión de los resultados mediante un análisis comparativo con datos nacionales y estatales. El objetivo del documento es presentar los indicadores de pobreza para las zonas rurales de Zacatecas, de forma que se localicen las causas de esta pobreza para cada región, causas que se asocian con diferentes dimensiones de las vidas de las personas.

PALABRAS CLAVE: pobreza rural, Zacatecas, pobreza multidimensional, vulnerabilidad por carencias sociales, marginación.

* Estudiante del Doctorado en Estudios del Desarrollo de la Universidad Autónoma de Zacatecas, México.

** Decente-investigador de la Unidad Académica de Economía de la Universidad Autónoma de Zacatecas, México. 
ABSTRACT: This article presents the results of a measurement of rural poverty in the state of Zacatecas, based upon a survey designed with that goal in mind. The data is from 2009, and the survey employed a multidimensional focus proposed by Coneval. The article is divided into three parts: in the first, the concept of poverty is discussed; in the second, relevant data is presented on poverty and population at the national level and in Zacatecas; in the third, the results of the measurement of rural poverty in the state is analyzed. The article discusses the results of the study through a comparative analysis with national and state data. The goal of this work is to present poverty indicators for the rural areas of Zacatecas in a manner that identifies the causes of this poverty for each region; causes which are associated with the various dimensions of people's individual lives.

KEY WORDS: rural poverty, Zacatecas, multidimensional poverty, sociallydisadvantaged vulnerability, marginalization. 


\section{INTRODUCCIÓN}

7 a pobreza, sobre todo la extrema, es un fenómeno eminentemente rural: en ese ámbito se ubican tres cuartas partes de la población mundial. Estudiar ese problema es fundamental para formular cualquier estrategia de desarrollo que pretenda reducirlo. En América Latina los actuales niveles de pobreza corresponden a los de hace casi 20 años, lo cual muestra que el flagelo no cede ante las políticas sociales diseñadas para superarlos. La informalidad y la precariedad en el empleo son factores clave de la pobreza rural, según Klein (2012). El hecho de que la población tienda a concentrarse cada vez más en localidades urbanas ha generado un desinterés por los espacios rurales, especialmente en el diseño de políticas que atiendan la especificidad de la problemática.

Zacatecas es una entidad con un alto índice de ruralidad. Para 2010, según datos del censo, la población residente en localidades con menos de 2,500 habitantes llegaba a 40.5 por ciento (604,070 individuos). En ese año era la sexta entidad del país con mayor población rural y la sexta con mayor pobreza, esto último según el Consejo Nacional de Evaluación de la Política de Desarrollo Social (Coneval). Por ello se afirma con frecuencia que en Zacatecas existe una extensa pobreza rural que tiende a asociarse con alta marginación, bajo desarrollo humano y emigración. Se supone muy extendida, pero hasta el momento no se ha calculado su dimensión.

El objetivo del documento es presentar los indicadores de pobreza rural de Zacatecas con base en una encuesta levantada para tal fin y la metodología multidimensional propuesta por el Coneval. El total de hogares entrevistados fue de 1,162 elegidos de manera aleatoria; el tamaño de la muestra fue determinado mediante un esquema de muestreo estratificado y por conglomerados. ${ }^{1}$ Por razones de espacio se omiten las características de la muestra y su diseño.

${ }^{1}$ La muestra fue diseñada por el actuario Jaime Mojica del INEGI, le agradecemos el apoyo brindado. 
El trabajo se conforma por tres apartados. En el primero se discute el concepto de pobreza; en el segundo se presentan datos relevantes sobre pobreza y población a nivel nacional y para Zacatecas; en el tercero se analizan los resultados de las mediciones de pobreza rural en la entidad.

\section{CONCEPTO DE POBREZA}

En América Latina, los primeros estudios para analizar el problema fueron los referidos a la marginación, concepto muy relacionado al de pobreza. La marginación se refiere a las personas cuyo acceso a educación, servicios de salud, empleo formal y condiciones de vivienda digna es limitado, precario o nulo (Kay, 2006). Los dos enfoques que se desarrollaron sobre marginación en América Latina se inspiraron en las teorías de la modernización y la marxista. Kay (2006) indica que el enfoque basado en la primera considera que la marginación surge de la falta de participación e integración de algunos individuos y grupos al sistema económico, político y social. La población marginada es vista como responsable e incapaz de superar su situación de marginalidad, por lo que el gobierno debe diseñar programas especiales para facilitar su integración al proceso de modernización. Por su parte, desde la teoría marxista la marginación se debe a la forma en que los países en desarrollo se han integrado al sistema capitalista. Para Kay, este enfoque se ubica en la teoría de la dependencia. La «masa marginal», concepto creado por José Nun (Kay, 2006) emerge del proceso de «desarrollo dependiente», término forjado por Cardoso, o del «desarrollo del subdesarrollo», según la expresión de Gunder Frank, que crea una fuerza de trabajo excedente que el país dependiente es incapaz de absorber en el sector formal de la economía, lo cual lleva a la emergencia de un polo marginal, término de Quijano, o a lo que más tarde sería referido como un sector informal, que al ser incapaz de encontrar empleo formal tiene que crear sus propios mecanismos de sobrevivencia.

Lo relevante para los estudios sobre pobreza y marginación rural es que el enfoque de la modernización reduce éstas a atributos de grupos o individuos que los incapacita para participar en la vida económica, social, política

$136 \frac{\text { PRIMER SEMESTRE } 2015}{\text { MIGRACIÓN Y DESARROLLO NÚM. } 24}$ 
y cultural del país; el enfoque marxista-dependentista considera a la marginación como una condición estructural creada y reproducida por el actual sistema capitalista y el proceso de globalización (Kay, 2006).

Si nos apegamos a este segundo enfoque para abordar el problema de la pobreza rural, entonces aceptamos que se trata de un fenómeno estructural. Como señala Rubio (2004: 949) para el caso de México: la ruina productiva de los productores rurales de bienes básicos responde a una nueva forma de dominio impulsado por las transnacionales, una «subordinación desestructurante, en tanto que no permite reproducirlos y tiende a excluirlos de la esfera productiva».

A continuación se expondrán algunos conceptos de pobreza tanto de organismos que tienen a su cargo la medición, como de algunos teóricos destacados en el tema. Empecemos con los últimos.

De acuerdo con Spicker (2009), el concepto de pobreza se asocia directamente con cuatro condiciones: la material, que se refiere a la falta de recursos para alcanzar un nivel de vida digno, y que a su vez puede ser entendida mediante los conceptos de necesidad, patrón de privaciones y limitación de recursos; la económica, que se relaciona directamente con el ingreso, mediante los conceptos de nivel de vida, desigualdad y posición económica; la social, la cual refleja el modo en que vive la población de acuerdo con la clase social, el grado de dependencia, la carencia de seguridad básica, la ausencia de titularidades y la exclusión; y una última, que se denomina condición moral, que expresa el grado de aceptabilidad o inaceptabilidad de las carencias que conlleva la pobreza. Estas definiciones tienen la característica de que son distintas, específicas, se superponen y también se vinculan entre sí.

Por su parte, Townsend (citado por Boltvinik, 2003: 708) dice:

La pobreza se puede definir de manera objetiva y aplicarse de modo consistente sólo en términos del concepto de privación relativa [...]. El término se entiende de manera objetiva y no subjetivamente. Se puede decir que los individuos, las familias y los grupos de la población se encuentran en pobreza cuando carecen de los recursos para obtener los tipos de dietas, participar en las actividades y tener las condiciones de vida y las comodidades que se acostumbran, o que al 
menos son ampliamente promovidos o aprobados, en las sociedades a las que pertenecen. Sus recursos están tan significativamente por debajo de los del individuo o la familia promedio que resultan, en efecto, excluidos de los patrones ordinarios de vida, costumbres y actividades.

De particular importancia es la definición de Sen (2000: 114):

La pobreza debe concebirse como la privación de las capacidades básicas y no meramente como la falta de ingresos, que es el criterio habitual con el que se identifica la pobreza. La perspectiva de la pobreza basada en las capacidades no entraña el rechazo de la razonable idea de que la falta de renta (ingreso) es una de las principales causas de la pobreza, ya que la falta de renta puede ser una importante razón por la que una persona está privada de capacidades.

Entre tales capacidades, sobresalen la perspectiva de una vida larga sin padecer enfermedades evitables, así como la posibilidad de adquirir conocimientos para articular las habilidades de reflexión y comprender el entorno en que se vive.

Existe una discusión teórico-conceptual acerca de qué criterio habrá de emplearse para definirla, la propuesta por Townsend, la relativa, o la de Sen, la absoluta. La discusión entre Townsend y Sen gira en torno a cómo considerar pobre a un individuo: el primero afirma que se encuentra en pobreza si no cuenta con los recursos suficientes para acceder a las condiciones de vida, costumbres y actividades, tomando en cuenta el tiempo y el lugar, es decir, que los satisfactores necesarios para la vida no son iguales en todas las sociedades y que la pobreza depende del nivel general de riqueza. En cambio, Sen sostiene que «existe un núcleo irreductible en la idea de pobreza. Si hay evidencias de hambre e inanición, entonces hay pobreza, independientemente de cualquier situación relativa» (Sen, citado por Spicker, 2009); las necesidades de cada sociedad pobre son las mismas, y no tienen una dependencia ni del tiempo ni del espacio, sino que la pobreza depende del nivel general de riqueza.

Gordon (2004) afirma, por su parte, que la pobreza es un fenómeno que causa daño social y que hace que los individuos sufran de inanición, carezcan

$138 \frac{\text { PRIMER SEMESTRE } 2015}{\text { MIGRACIÓN Y DESARROLLO NÚM. } 24}$ 
de agua potable, servicios sanitarios, salud y educación, todo esto provocado por algo que denomina "una opción política», en referencia a que no es un hecho fortuito o inevitable, o que sea provocado por la falta de dinero, sino por la falta de voluntad política para acabarla.

Boltvinik (2003: 454) define a la pobreza como un fenómeno multidimensional, en el que el bienestar de los hogares depende de: 1) el ingreso corriente; 2) los activos no básicos y la capacidad de endeudamiento del hogar; 3) el patrimonio familiar, concebido como el conjunto de activos y bienes durables que proporcionan servicios básicos a los hogares (vivienda y equipamiento doméstico básico); 4) el acceso a bienes y servicios gratuitos (consumo público); 5) los conocimientos de las personas, considerados no como medio para la obtención de ingresos, sino como satisfactores directos de la necesidad humana de entendimiento, y 6) el tiempo libre disponible. Siendo pobre aquel que carece de una o más fuentes de bienestar.

Puede apreciarse que la pobreza es un concepto con definiciones diferentes. Cada autor emite o elige una definición, pero en general todos coinciden en que se refiere a garantizar el bienestar social de las personas. Aunque el significado de pobreza, generalmente, se dirige a considerar los aspectos materiales que permiten desarrollar la vida y se mide de una forma indirecta mediante el ingreso monetario, en la actualidad los métodos de medición más completos son los que toman en cuenta, además, indicadores que tienen que ver con garantizar un nivel de vida digno.

Enseguida se aborda la definición de pobreza según algunas instituciones.

La Comisión Económica para América Latina y el Caribe (CEPAL) la define como un síndrome situacional en el que se asocian el infraconsumo, la desnutrición, las precarias condiciones de vivienda, los bajos niveles educacionales, las malas condiciones sanitarias, una inserción inestable en el aparato productivo, actitudes de desaliento y anomia, poca participación en los mecanismos de integración social, y quizá la adscripción a una escala particular de valores diferenciada en alguna medida de la del resto de la sociedad, como una situación que expresa la carencia de recursos económicos o de condiciones de vida que la sociedad considera básicos, de acuerdo con normas sociales de referencia que reflejan derechos sociales mínimos y objetivos 
públicos. Estas normas se expresan en términos tanto absolutos como relativos, y son variables en el tiempo y los diferentes espacios nacionales (Hopenhayan, 2003).

Para el Banco Mundial,

la pobreza es hambre; es la carencia de protección; es estar enfermo y no tener con qué ir al médico; es no poder asistir a la escuela, no saber leer, no poder hablar correctamente; no tener un trabajo; es tener miedo al futuro, es vivir al día; la pobreza es perder un hijo debido a enfermedades provocadas por el uso de agua contaminada; es impotencia, es carecer de representación y libertad (Romero, 2002: 88).

El Programa de Naciones Unidas para el Desarrollo (PNUD) en 1997 presentó por primera vez la expresión pobreza humana basada en el concepto de capacidades de Sen y la definió como la negación de opciones y oportunidades de vivir una vida tolerable (Mathus, 2008).

En México, los estudios oficiales sobre pobreza son importantes porque proporcionan información indispensable sobre la magnitud y distribución espacial. Éstos recibieron especial atención en el sexenio de José López Portillo. En 1977 se creó la Coordinación General del Plan Nacional de Zonas Deprimidas y Grupos Marginados (Coplamar). Los estudios coordinados por Coplamar sobre mínimos de bienestar incluyen la construcción de un «índice de marginación» basado en 19 indicadores. ${ }^{2}$ Con base en éste se determina el grado de marginación a nivel nacional, regional y municipal (González Montes, 1994). A partir de 1990, el Consejo Nacional de Población (Conapo) calcula los índices de marginación con base en la información generada por los Censos de Población y Vivienda y los conteos generales de 1995 y 2005 del Instituto Nacional de Estadística, Geografía e Informática (INEGI), considerando cuatro dimensiones: educación, vivienda, ingreso de la población ocupada y

2 Según este índice la marginación se expresa en: 1) bajos niveles de ingreso de la PEA; 2) altos niveles de subempleo; 3) altos porcentajes de población rural agrícola; 4) incomunicación de las localidades; 5) alimentación inadecuada; 6) bajos niveles de escolaridad; 7) bajos niveles de salud y dificultad de acceso a servicios médicos; 8) viviendas inadecuadas y sin servicios; 9) escaso acceso a otros satisfactores como calzado, radio y televisión.

$140 \frac{\text { PRIMER SEMESTRE } 2015}{\text { MIGRACIÓN Y DESARROLLO NÚM. } 24}$ 
la distribución territorial de la población, de donde se generan nueve indicadores. Los índices se presentan a nivel de entidad, municipio, localidad y áreas geoestadísticas básicas (AGEB) urbanas.

Las fuentes oficiales para medir la pobreza provienen de las Encuestas de Ingreso y Gasto de los Hogares, levantadas por el InEGI. Durante la administración de Vicente Fox (2000-2006), la Secretaría de Desarrollo Social (Sedesol) se propuso construir una medición oficial de la pobreza; en diciembre de 2003 se aprobaría la Ley General de Desarrollo Social, con la que se definió el marco para que fuese el Coneval quien definiera los lineamientos y criterios para la definición, identificación y medición de la pobreza.

Hasta 2007, en México el Coneval estuvo empleando tres líneas de pobreza: la alimentaria, la de capacidades y la patrimonial. El primer tipo es la que vive un hogar al que, aun dedicando todo su ingreso para la adquisición de alimentos, no le alcanza para cubrir el costo de dicha canasta. La segunda es la que padecen aquellos hogares que, comparando su ingreso con el costo de alimentos, educación y salud, éste resulta inferior. Finalmente, la última es la que padecen los hogares que aun destinando todo su ingreso no les alcanza para alimentos, salud, educación, vivienda, transporte y vestido. Sabemos que el gobierno está combatiendo la pobreza extrema y no la moderada, omisión que contribuye al aumento de la última. Es un caso de medición unidimensional de la problemática, al considerar al ingreso como el determinante del bienestar de las familias.

Hacia 2007, el Coneval modificaría el concepto adoptando el de pobreza multidimensional que refiere a que «una persona se encuentra en situación de pobreza multidimensional cuando no tiene garantizado el ejercicio de al menos uno de sus derechos para el desarrollo social, y si sus ingresos son insuficientes para adquirir los bienes y servicios que requiere para satisfacer sus necesidades» (Coneval, 2010: 38).

La pobreza, de acuerdo con el Coneval, se desagrega según la situación de la población en pobreza moderada y extrema; conforme a su vulnerabilidad: por carencias sociales (rezago educativo, carencias de acceso a los servicios de salud, seguridad social, calidad y espacios de la vivienda, servicios básicos en la vivienda, y alimentación) y por ingresos. La identificación de 
la población en situación de pobreza se realiza en dos etapas: en la primera, se determina si los ingresos de una persona son insuficientes para la satisfacción de sus necesidades y si padece al menos una de las seis carencias anteriormente mencionadas; $y$, en la segunda, se combinan los indicadores generados en la etapa previa, con el fin de identificar a la población en situación de pobreza multidimensional. Cualquier persona podrá ser clasificada en uno y sólo uno de los siguientes cuatro cuadrantes:

I. Pobres multidimensionales. Individuos con ingreso inferior al valor de la línea de bienestar y que padecen al menos una carencia social.

II. Vulnerables por carencias sociales. Aquel que presenta una o más carencias sociales, pero cuyo ingreso es superior a la línea de bienestar.

III. Vulnerables por ingresos. Quien que no presenta carencias sociales y cuyo ingreso es inferior o igual a la línea de bienestar.

IV. No pobre multidimensional y no vulnerable. Población cuyo ingreso es superior a la línea de bienestar y que no tiene carencia social alguna (Coneval, 2010: 42).

La modificación referida representa un avance en cuanto a su carácter multidimensional, pero no se puede negar que subestima las cifras. Esto ocurre al considerar pobres a las personas con un ingreso inferior a la línea del bienestar y que sufren al menos una carencia, o cuando denomina pobres extremos a quienes tengan un nivel de ingreso inferior a la línea de bienestar mínimo y padezcan tres carencias, de tal forma que una persona con dos carencias (aunque sean alimentarias o de salud) no es considerada un pobre extremo, además sigue comparando ingreso corriente total con el costo de la canasta alimentaria, a pesar de ser imposible que un hogar pueda dedicar todo su ingreso para alimentarse, puesto que tiene que satisfacer otras necesidades de igual importancia, como la salud o el aseo personal.

Como ya se indicó anteriormente, esta metodología es la usada en el presente trabajo. Antes de pasar a los resultados, se expone brevemente la importancia de la población rural en México y en Zacatecas, y la incidencia de la pobreza en estos niveles.

$142 \frac{\text { PRIMER SEMESTRE } 2015}{\text { MIGRACIÓN Y DESARROLLO NÚM. } 24}$ 


\section{Población rural y pobreza en México y Zacatecas}

El reciente interés por el desarrollo en el ámbito rural se debe principalmente a que es ahí en donde se concentra la mayoría de la población que vive en condiciones de pobreza y marginación. En el caso de América Latina, según datos de la CEPAL, para 201052.6 por ciento de la población rural vivía en condiciones de pobreza y 30 por ciento en pobreza extrema.

En 2010, México tenía 26 millones de personas (23 por ciento) residiendo en localidades rurales. Los datos sobre pobreza medida según el enfoque multidimensional propuesto por el Coneval indican que 64.9 por ciento de ésta vivía en condiciones de pobreza y 23.9 por ciento, en pobreza extrema. A pesar de los programas focalizados para superarla, el flagelo persiste, pues se trata de un problema estructural profundizado debido a las políticas de corte neoliberal implementadas desde la década de los ochenta.

Según Tetreault (2012), dichas políticas se tradujeron en la desaparición de programas de desarrollo iniciados en la década anterior, como el Programa Integral para el Desarrollo Rural (Pider), la Coplamar y el Sistema Alimentario Mexicano (SAM), así como la reducción de aranceles y cuotas para las importaciones agrícolas, que culminaría después en el Tratado de Libre Comercio de América del Norte (TLCAN). Se suprimieron o privatizaron organismos gubernamentales vinculados al sector agrícola y se eliminaron precios de garantía y diversos subsidios; todo esto llevó a la caída vertiginosa de los precios del sector en la década de los noventa, el aumento de las importaciones provenientes de Estados Unidos y, al mismo tiempo, el aumento en el costo de los insumos para los agricultores.

El resultado de lo anterior fue el aumento de la pobreza, al grado de preocupar a los propios organismos multinacionales encargados de promover dicho modelo. Fueron ellos quienes recomendaron políticas focalizadas en los pobres, centradas en la transferencia de recursos monetarios. En el caso particular de México, éstas ahora se cristalizan bajo el Programa Oportunidades, antes Progresa, implementado desde 1997 durante el gobierno de Ernesto Zedillo. No obstante, después de 15 años del programa, la pobreza persiste en sus manifestaciones más agudas, como la alimentaria. 
En cuanto a Zacatecas, esta entidad se caracteriza por tener un alto porcentaje de población rural. Para 2010 es la sexta con mayor población que reside en localidades con menos de 2,500 habitantes. Lo que caracteriza a la población rural es su dispersión en un gran número de localidades, esto complica su acceso a mercados de trabajo y de servicios, y justifica el abandono del gobierno argumentando altos costos para dotarles de los servicios básicos que constitucionalmente tiene obligación de proveer.

Para 2000, 46.7 por ciento de la población del estado residía en localidades rurales, en 2010 el porcentaje se redujo a 40.5 por ciento. Las localidades urbanas (con más de 15 mil habitantes) concentran para este último año un porcentaje similar al de las rurales (40.2 por ciento). Véase cuadro 1.

\section{CUADRO 1}

Zacatecas, distribución de la población según tamaño de localidad, 2000-2010

\begin{tabular}{|c|c|c|c|c|}
\hline \multirow[b]{2}{*}{$\begin{array}{c}\text { TIPO DE } \\
\text { LOCALIDAD }\end{array}$} & \multicolumn{4}{|c|}{2000} \\
\hline & LOCALIDADES & $\%$ & $\begin{array}{c}\text { POBLACIÓN } \\
\text { TOTAL }\end{array}$ & $\%$ \\
\hline Rural & 4,821 & 98.8 & 631,546 & 46.7 \\
\hline Semirural & 33 & 0.7 & 114,648 & 8.5 \\
\hline Semiurbana & 18 & 0.4 & 153,135 & 11.3 \\
\hline Urbana & 10 & 0.2 & 454,281 & 33.6 \\
\hline \multirow[t]{2}{*}{ Total } & 4,882 & 100.0 & 1'353,610 & 100.0 \\
\hline & \multicolumn{4}{|c|}{2010} \\
\hline Rural & 4,605 & 98.6 & 604,070 & 40.5 \\
\hline Semirrural & 33 & 0.7 & 114,214 & 7.7 \\
\hline Semiurbana & 22 & 0.5 & 173,875 & 40.2 \\
\hline Urbana & 12 & 0.3 & 598,509 & 40.2 \\
\hline Total & 4,672 & 100.0 & 1'490,668 & 100.0 \\
\hline
\end{tabular}

Rural: Menos de 2,500 habitantes; Semirrural: de 2,500 a 4,999 habitantes; Semiurbana: de 5,000 a 14,999 habitantes; Urbanas: más de 15,000 habitantes.

Fuente: Elaborado con base en Censos de Población y Vivienda.

Aunque gran parte de la población de la entidad se ubica en zonas rurales, el sector agropecuario ha ido perdiendo importancia, en 2010 aportaba

$144 \frac{\text { PRIMER SEMESTRE } 2015}{\text { MIGRACIÓN Y DESARROLLO NÚM. } 24}$ 
8.9 por ciento del producto interno bruto (PIB). En el nivel nacional los hogares rurales dependen cada vez más de ingresos no agropecuarios para subsistir; en Zacatecas las remesas internacionales se han convertido en una fuente preponderante del ingreso en el medio rural. Según cálculos oficiales, para 2010 la entidad tiene el más alto índice de intensidad migratoria internacional y el mayor porcentaje de hogares receptores de remesas (11.4 por ciento; en 2000 eran de 13 por ciento).

La crisis de las actividades agropecuarias y la debilidad del resto de los sectores económicos traen como consecuencia un mercado laboral regional poco dinámico, incapaz de absorber la población excedente del campo y han generado una fuerte expulsión de población a otras entidades del país y, sobre todo, a Estados Unidos. Hasta 2005, Zacatecas se caracterizaba por ser una de las entidades con menor tasa de crecimiento demográfico a nivel nacional.

\section{GRÁFICA 1}

Zacatecas: población en pobreza, 2008-2010³ (\%)

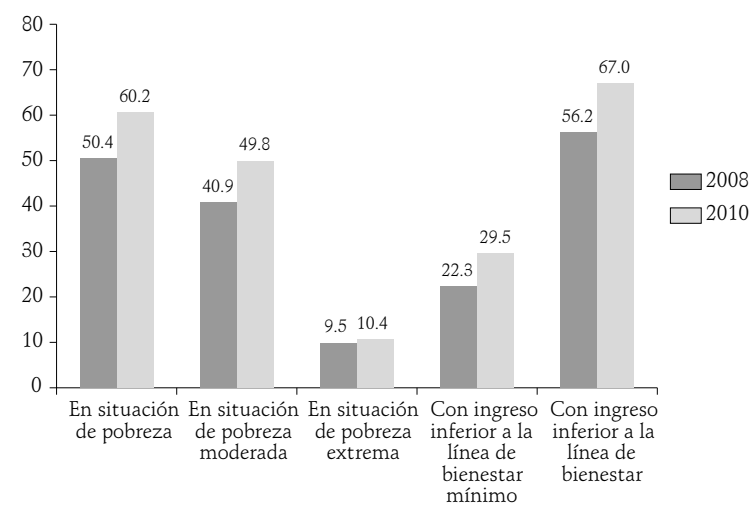

Fuente: Elaborado con base en datos en línea disponibles en www.coneval.gob.mx

3 Para 2010 la líneas de bienestar (equivalentes a las líneas de pobreza) que ayudan a clasificar a la población en pobreza y pobreza extrema, fueron, la mínima de 697.93 pesos per cápita mensuales para las localidades rurales, y de 990.77 pesos para las localidades urbanas. La de bienestar fue de 1,348.62 y 2,140.05 pesos para el medio rural y urbano, respectivamente. El lector interesado puede consultar la metodología en www.coneval.gob.mx 
No disponemos de datos de pobreza por localidad para Zacatecas, por lo que revisaremos la incidencia a nivel estatal. Para 2010, 60.2 por ciento de la población del estado vivía en condiciones de pobreza. En dos años (20082010) su incidencia aumentó 10 puntos porcentuales, lo que la ubicó como la de mayor crecimiento en el número de pobres. Esto último asociado con la caída de las remesas provenientes de Estados Unidos, pero también con el mal temporal para las actividades agrícolas, que de paso ha afectado a la ganadería, otra importante actividad económica del estado (véase gráfica 1).

\section{Pobreza rural en Zacatecas}

En este apartado se presentan los resultados de la encuesta a hogares rurales levantada entre junio y diciembre de 2010 en localidades rurales de Zacatecas. La información fue captada en 2009. Los resultados que se presentan se refieren a la muestra, 1,162 hogares, 4,803 personas. La muestra tiene representatividad para las localidades menores a 2,500 habitantes. La metodología aplicada es la recomendada por el Coneval bajo el enfoque multidimensional, la única diferencia es que usamos gasto corriente en lugar del ingreso. La variable de gasto corriente se construyó según criterios indicados en la Encuesta Nacional de Ingreso y Gasto de los Hogares (ENIGH, 2010): gasto corriente monetario más gasto corriente no monetario, excluyendo, como lo hace el Coneval, los rubros de autoconsumo, los regalos recibidos una sola vez durante el año y el costo imputado por alquiler de la vivienda. ${ }^{4}$ Asimismo, se determinó excluir del gasto corriente monetario los robos y pérdidas de dinero, puesto que se consideran gastos extraordinarios para los hogares. En la encuesta hubo casos de extorsiones y los hogares tuvieron que vender activos o recurrir a familiares en el exterior para poder cubrir los pagos exigidos. El criterio para excluirlos fue el señalamiento en la metodología del Coneval, que indica que se deben incluir aquellas entradas que le

${ }^{4}$ Esto se verificó en los archivos disponibles para el cálculo de la pobreza en sPss, que se pueden bajar de la página web del Coneval.

$146 \frac{\text { PRIMER SEMESTRE } 2015}{\text { MIGRACIÓN Y DESARROLLO NÚM. } 24}$ 
permiten a los hogares obtener los satisfactores que requieren sin disminuir los bienes o activos que poseen. La ventaja de medir la pobreza a través del gasto y no del ingreso es que el primero se percibe como un método directo, que mide el nivel de consumo efectivo realizado por los hogares. Se cuenta con datos para medir el ingreso del hogar, pero hacerlo por esa vía es más complejo dadas las características de la encuesta, esta tarea se ha dejado para una fecha posterior y tendremos la oportunidad de comparar resultados.

El gasto corriente fue deflactado para ponerlo a precios de julio de 2009 y se usaron las líneas de bienestar actualizadas por el Coneval, promedio 2009. Los cálculos per cápita se hicieron ajustando el tamaño del hogar por economías de escala y escalas de adulto equivalente, según propone el mismo Consejo.

El cuadro 2 muestra los resultados de las mediciones sobre pobreza rural de la encuesta, última columna. Se incluyen los datos a nivel nacional (total y para localidades rurales) y el total estatal, con el fin de comparar. Para 2009, 61 por ciento de la población rural del estado se encontraba en pobreza, es decir, no tenía garantizado al menos uno de sus derechos sociales y su gasto fue menor a la línea de bienestar. Este indicador es cercano al total estatal y superior al nacional, pero está por debajo del correspondiente a localidades rurales a nivel nacional (64.9 por ciento). En cambio, el indicador de pobreza moderada en el medio rural de Zacatecas es parecido a nivel nacional, lo mismo pasa con la pobreza extrema, aunque en las zonas rurales del estado es ligeramente inferior al total rural nacional.

De la comparación entre la incidencia total y rural a nivel nacional y los datos rurales a nivel estatal, podemos captar las diferencias en la composición de pobreza en zonas rurales: mientras que la pobreza moderada en promedios nacionales y estatales triplica o cuadruplica a la extrema, en zonas rurales la diferencia entre una y otra no es tan grande, es menos del doble, es decir, en las zonas rurales la severidad de la pobreza es muy superior a las no rurales. Recordemos que, según el enfoque multidimensional, una persona se encuentra en pobreza extrema cuando presenta al menos tres carencias sociales y su ingreso es inferior a la línea de bienestar mínimo, y está en pobreza moderada cuando presenta menos de tres carencias sociales y su ingreso es superior a la línea de bienestar mínimo pero inferior a la línea de bienestar. 


\section{CUADRO 2}

Pobreza multidimensional: Comparación de resultados de la encuesta a hogares rurales 2009 y datos del Coneval, 2010 (\% de personas)

\begin{tabular}{|c|c|c|c|c|}
\hline \multirow[b]{2}{*}{ INDICADORES } & \multicolumn{3}{|c|}{ CONEVAL } & \multirow{2}{*}{$\begin{array}{c}\text { EHRURZAC } \\
\text { ZAC RURAL } \\
2009\end{array}$} \\
\hline & $\begin{array}{l}\text { NACIONAL } \\
2010\end{array}$ & $\begin{array}{l}\text { RURAL NAC } \\
2010\end{array}$ & $\begin{array}{l}\text { ZACATECAS } \\
2010\end{array}$ & \\
\hline \multicolumn{5}{|l|}{ Pobreza } \\
\hline Población en situación de pobreza & 46.2 & 64.9 & 60.2 & 61.0 \\
\hline En pobreza moderada & 35.8 & 40.9 & 49.8 & 39.9 \\
\hline En pobreza extrema & 10.4 & 23.9 & 10.4 & 21.2 \\
\hline Población vulnerable por carencias sociales & 28.7 & 28.8 & 18.7 & 35.5 \\
\hline Población vulnerable por ingresos & 5.8 & 1.1 & 6.8 & 0.0 \\
\hline Población no pobre y no vulnerable & 19.3 & 5.2 & 14.3 & 3.5 \\
\hline \multicolumn{5}{|l|}{ Privación Social } \\
\hline Población con al menos una carencia & 74.9 & 93.6 & 78.9 & 96.5 \\
\hline Población con al menos tres carencias sociales & 26.6 & 50.3 & 22.8 & 62.0 \\
\hline \multicolumn{5}{|l|}{ Indicadores de carencia social } \\
\hline Rezago educativo & 20.6 & 33.9 & 22.8 & 33.9 \\
\hline Carencia por acceso a servicios de salud & 31.8 & 32.2 & 27 & 30.5 \\
\hline Carencia por acceso a la seguridad social & 60.7 & 81.9 & 66.4 & 85.3 \\
\hline Carencia por calidad y espacios de la vivienda & 15.2 & 29.2 & 5.9 & 41.7 \\
\hline $\begin{array}{l}\text { Carencia por acceso a los servicios básicos en } \\
\text { la vivienda }\end{array}$ & 16.5 & 46.6 & 16.1 & 46.2 \\
\hline Carencia por acceso a la alimentación & 24.9 & 33.6 & 24.8 & 59.6 \\
\hline \multicolumn{5}{|l|}{ Bienestar } \\
\hline $\begin{array}{l}\text { Población con un ingreso inferior a la línea de } \\
\text { bienestar mínimo }\end{array}$ & 19.4 & 35 & 29.5 & 23.1 \\
\hline $\begin{array}{l}\text { Población con un ingreso inferior a la línea de } \\
\text { bienestar }\end{array}$ & 52 & 66 & 67.0 & 61.0 \\
\hline
\end{tabular}

Fuente: Coneval (2011) y Encuesta a Hogares Rurales de Zacatecas, 2009.

Nota: Los datos de la Ehrurzac se refieren a la población de la muestra, 4,803 personas en total.

La metodología permite identificar a dos grupos de población vulnerable: por carencias sociales y por ingresos. En el primero estaría la población que tiene un ingreso superior a la línea de bienestar pero presenta una o más carencias sociales; en el segundo, quienes no presentan carencias sociales y cuyo ingreso es inferior o igual a la línea de bienestar. El porcentaje de población vulnerable a carencias sociales en el medio rural de Zacatecas es

$148 \frac{\text { PRIMER SEMESTRE } 2015}{\text { MIGRACIÓN Y DESARROLLO NÚM. } 24}$ 
superior al nacional, también está muy por encima del promedio estatal. En los indicadores de privación social, vemos cómo 96.5 por ciento de la población padece al menos una carencia social, indicador también por encima del resto de los datos sujetos a comparación.

Los indicadores de privación social de las localidades rurales de Zacatecas son muy cercanos a los nacionales, excepto en dos: carencia por calidad y espacios de la vivienda y por acceso a alimentación. Este último se obtuvo según la medida complementaria propuesta por el Coneval: comparar el gasto de alimentos per cápita con el costo de la canasta básica alimentaria. El resultado indica que 59.6 por ciento de las personas tiene un gasto en alimentos menor a la línea de bienestar mínimo. Se procedió de esta manera, pues en la encuesta no se incluyó el conjunto de preguntas usado para medir dicha carencia. Digamos que la medida complementaria es mejor que la medición usada por el Coneval, con la acotación de que sería más precisa, y seguramente menor aunque no tenemos la certeza sobre la magnitud, si se incluyera el valor de los alimentos producidos y consumidos en el hogar, el autoconsumo. Como ya se indicó anteriormente, éste no fue considerado, puesto que tampoco el Coneval lo incluye en el ingreso corriente de los hogares. En el caso de calidad y espacios de la vivienda, sorprende el resultado tan alto; se recalculó para verificar que no hubiera errores y el resultado fue el mismo. Aquí la única diferencia con respecto al Coneval es que para medir el hacinamiento, indicador que resultó más alto en esta dimensión, se tomó el número de cuartos usados para dormir en vez del total de cuartos en la vivienda. A nivel de hogares, 33 por ciento presenta carencias por calidad y espacios de la vivienda, a nivel de personas se eleva considerablemente hasta alcanzar 41.7 por ciento, indicado en el cuadro 2, muy por encima del nivel que muestran los promedios nacionales y estatales. En campo se constataron situaciones graves de hacinamiento, especialmente en las localidades con alto y muy alto grado de marginación social. Al respecto, tampoco estamos de acuerdo con las normas establecidas por el Coneval, pues en el caso del material de techos de las viviendas, todas superan la condición de carencia si su techo es diferente al material de desecho. En Zacatecas abundan las viviendas con techo de terrado con viguería realmente precarias, que no 
garantizan la protección de sus moradores en tiempos de lluvia, incluso los techos con lámina de cartón se consideran suficientes para superar esta carencia. De este modo, se asume que un muy alto porcentaje de población tiene viviendas adecuadas, cuando la realidad es distinta. Un programa de vivienda rural es urgente, para que las familias tengan un espacio adecuado para su convivencia. La antigüedad de la vivienda no se toma en cuenta, los muros pueden estar derrumbándose, lo mismo se puede decir de los techos, pero sólo importa el tipo de material. Esto sin mencionar aspectos subjetivos como el nivel de satisfacción de las personas, especialmente de las mujeres, con la casa que habitan. Esto se señala porque muchas mujeres comentaron que su casa era incómoda y quisieran tener mejores muebles, más cómodos, en donde realmente pudieran descansar.

En fin, las mediciones de pobreza son muy vulnerables a los umbrales que se establezcan. A pesar de ser un problema social importante, quizá el acento no debiera ponerse tanto en medirla, sino en superarla; en las discusiones sobre pobreza hay mucho sobre medición y menos sobre lo segundo. Educación, alimentación, acceso a servicios de salud y una vivienda digna pueden ser los elementos más importantes y puntos de partida para superar este problema tan relacionado con la dignidad humana y con la justicia social. El ingreso seguirá siendo importante en la medida en que el mercado sea el medio para obtener los satisfactores, es decir, mientras las relaciones capitalistas sean las que predominen, por lo que también se deben buscar mecanismos para una integración más justa de los pequeños productores, capacitación para el trabajo, uso eficiente de los recursos y aumento de la productividad.

Al final del cuadro se muestran los indicadores de bienestar, relacionados con el ingreso (para Zacatecas rural con el gasto). La población con gasto inferior a la línea de bienestar mínimo es 23.1 y 61 por ciento, menor al promedio estatal y al de zonas rurales del país. Es importante comentar algo en lo que Boltvinik insiste, para medir la pobreza extrema es incorrecto comparar el costo de la canasta alimentaria con el ingreso total del hogar, puesto que las personas además de alimentos tienen que adquirir otros satisfactores de igual importancia para la supervivencia, como salud e incluso

$150 \frac{\text { PRIMER SEMESTRE } 2015}{\text { MIGRACIÓN Y DESARROLLO NÚM. } 24}$ 
educación. Como ya se indicó, al comparar el gasto en alimentación con la línea de bienestar mínimo resulta que 59.6 por ciento de los hogares tiene un gasto en alimentos inferior a dicha línea, al compararlo con su gasto total sólo 23.1 por ciento estaría en pobreza extrema por ingresos, pero en nuestro caso dicho gasto incluye educación, salud, servicios para la vivienda, transporte y artículos para el aseo y cuidado personal, principalmente. Al usar el gasto corriente en lugar del ingreso corriente, es más evidente lo ilógico que resulta comparar ingreso total con costo de la canasta alimenticia.

La gráfica 2 presenta una mirada rápida sobre la situación de pobreza multidimensional para las localidades rurales. Los datos de la población en dicha situación ya fueron comentados. El grupo de no pobres puede estar en condiciones de vulnerabilidad por carencias sociales, es decir, con ingreso superior a la línea de bienestar pero con alguna carencia en derechos sociales, en esa posición estaría la mayoría de los no pobres (35.5 por ciento), vulnerabilidad por ingreso, es decir, con todos sus derechos sociales cubiertos pero con ingreso inferior a la línea de bienestar, sólo una persona de la muestra estaba en esta situación, pues como ya se comentó, la vulnerabilidad por carencias es especialmente aguda en el ámbito rural. Sólo 3.5 por ciento de la población de la muestra (de un total de 4,803 personas) resultó no pobre y no vulnerable, porcentaje menor al rural nacional (5.2 por ciento).

En el cuadro 3 se presentan los indicadores de profundidad e intensidad de la pobreza. Una vez identificados los individuos, se debe conocer qué tan pobres son. El Coneval recomienda dos medidas de profundidad, una en el espacio del bienestar, para lo cual se usa el índice FGT (Foster, Greer y Thorbecke), que mide la distancia promedio del ingreso de la población con un ingreso inferior a la línea de bienestar respecto a esta misma línea; la otra es sobre el índice de privación social a través de la proporción promedio de carencias sociales. La intensidad se obtiene multiplicando una medida de incidencia por una de profundidad, según propuestas hechas por Alkire y Foster (2007, citados en Coneval, 2010). Se proponen, de conformidad con el marco conceptual de la institución, las tres medidas indicadas en el cuadro 3, las cuales se comentan enseguida. 


\section{GRÁFICA 2}

Distribución de la población rural de Zacatecas según situación de pobreza, 2009

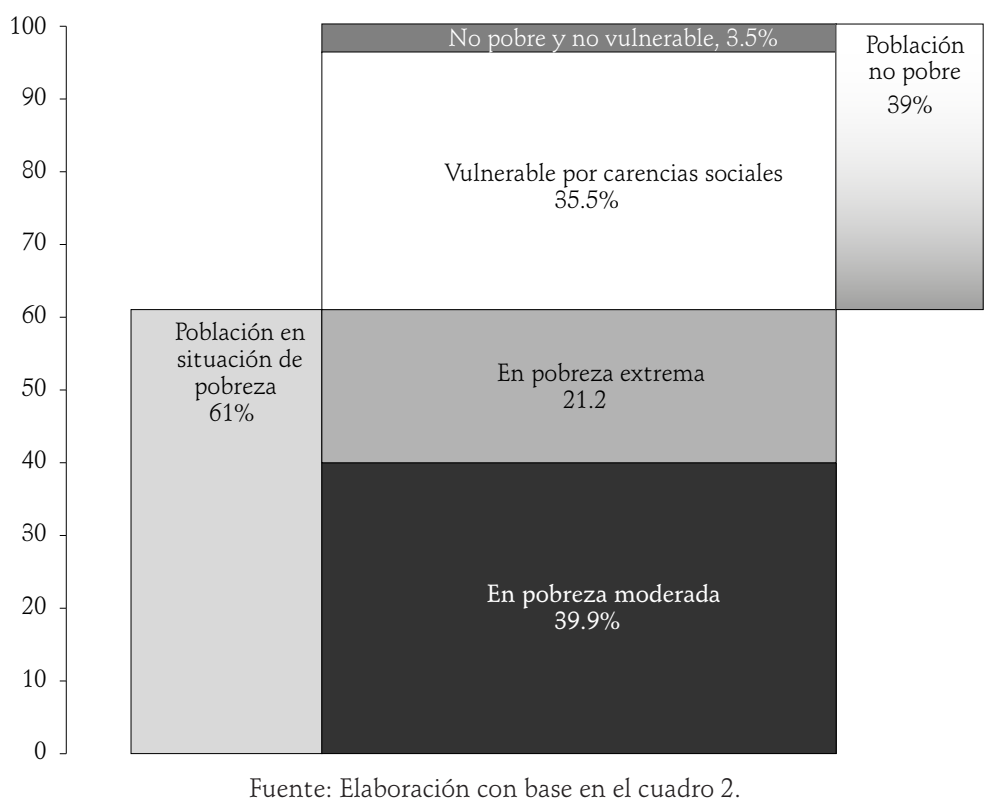

No sólo en el medio rural viven las personas más pobres, también las más vulnerables y carentes de sus derechos sociales básicos. El número promedio de carencias sociales de la población que vive en zonas rurales es mayor a los promedios nacionales, esto no es nuevo. Aunque estén acostumbrados, los habitantes rurales tienen derecho a mejor educación, servicios de salud y seguridad social, una vivienda digna y, desde luego, alimentación. Heredera de condiciones realmente precarias, la gente a veces no percibe estas carencias como injustas. Muchos sienten que su situación es mejor que la de sus padres, comparado en el tiempo quizá tengan razón, antes no tenían luz, agua en sus viviendas, se transportaban en el lomo de mulas, no había escuela; ésos fueron los principales argumentos señalados por los y las entrevistados/as cuando se les cuestionó al respecto; no obstante, sus parámetros de referencia deben ser sus contemporáneos y sus derechos humanos y civiles. 
El número de carencias promedio de la población rural en Zacatecas es de 3 (50 por ciento de los derechos sociales reconocidos por el Coneval), de la población en pobreza es de 3.6; la población en pobreza extrema padece 4.3 carencias en promedio, lo que significa que carece de cerca de 72 por ciento de los derechos sociales. En todos los casos, los indicadores de profundidad de privaciones sociales para Zacatecas son mayores a los rurales nacionales, salvo en la población vulnerable por carencias sociales (es decir, con ingreso superior a la línea de bienestar), en ese caso, el promedio en ambos rubros es de 2.2 (véase cuadro 3).

\section{CUADRO 3}

Indicadores de intensidad y profundidad de la pobreza, nacional y para localidades rurales de Zacatecas

\begin{tabular}{lccc}
\hline \multicolumn{1}{c}{ INDICADORES } & NACIONAL & RURAL NAC & EHRURZAC \\
& 2010 & 2010 & 1009 \\
\hline $\begin{array}{l}\text { Profundidad de la privación social } \\
\text { Población total }\end{array}$ & 1.7 & nd & 3.0 \\
Población en situación de pobreza & 2.5 & 3.0 & 3.6 \\
$\quad$ Población en situación de pobreza moderada & 2.1 & 2.5 & 3.2 \\
$\quad$ Población en situación de pobreza extrema & 3.7 & 3.9 & 4.3 \\
Población con al menos una carencia social & 2.3 & 2.8 & 3.1 \\
Población vulnerable por carencias sociales & 1.9 & 2.2 & 2.2 \\
Intensidad de la pobreza & & & \\
Población en situación de pobreza & 0.19 & 0.32 & 0.37 \\
Población en situción de pobreza extrema & 0.06 & 0.16 & 0.15 \\
Población con al menos una carencia social & 0.28 & 0.44 & 0.50 \\
\hline
\end{tabular}

${ }^{1}$ Se reporta el número promedio de carencias sociales del grupo de referencia.

${ }^{2}$ Corresponde al producto de la proporción de la población en el grupo de referencia y la proporción promedio del total de carencias que presenta el mismo grupo.

Fuente: Coneval (2012) y estimaciones con base en la Encuesta a Hogares Rurales de Zacatecas.

La intensidad de la pobreza mide, como el nombre lo indica, qué tan pobres son los individuos. No es lo mismo sufrir una sola carencia social y contar con un ingreso ligeramente inferior a la línea de bienestar, que padecer seis carencias sociales y percibir un ingreso mucho menor al del umbral. De esta forma, el valor máximo que el coeficiente de intensidad puede alcanzar 
es el correspondiente al de incidencia $(\mathrm{H})$, es decir, proporción de población en pobreza, en nuestro caso, pobreza multidimensional; mientras más cercano sea con $\mathrm{H}$ el valor de intensidad, más pobres serán los sujetos en cuestión. Los cálculos de intensidad para la población rural de Zacatecas, calculados con base en la Ehrurzac, son superiores a los rurales nacionales. El índice de la población en situación de pobreza es de 0.37 , comparado con una incidencia de 0.61 , podemos hablar de una intensidad media alta, es decir, los pobres sufren más de 50 por ciento de carencias. La intensidad de la pobreza extrema es de 0.15 , comparada con una incidencia de 0.2 , lo cual nos indica que entre los pobres extremos la intensidad de la problemática es más alta que entre los pobres, con carencias mayores a 70 por ciento.

Siguiendo con la profundidad en el espacio del bienestar, el índice FGT para los pobres rurales de Zacatecas fue de 0.389 , lo que indica que en promedio, el ingreso (gasto en nuestro caso) de dichos sujetos se aleja en 39 por ciento de la línea de bienestar, de tal manera que si se quisiera eliminar la pobreza por ingresos, en promedio, se le tendrían que transferir 502 pesos mensuales a la población en esta situación. El índice para los pobres extremos es de 0.275 , de tal manera que si se quiere superar la pobreza extrema, se tendría que dar a quienes la padecen, en promedio, 165.5 pesos por mes, aunque eso sólo les permitiría dejar esa situación extrema. Bien podría ser combatida aumentando la frecuencia de las despensas con alimentos de mayor calidad, mismas que entraron en la medición del gasto corriente en nuestro caso. Esas despensas podrían incluir no sólo alimentos, sino también artículos para la higiene y cuidado personal.

El cuadro 4 muestra los indicadores correspondientes al contexto territorial. Se pusieron los datos a nivel nacional con el fin de tener un patrón de referencia. En cuanto a desigualdad, vemos que el índice de Gini para las localidades rurales de la muestra es mucho menor al nacional, menos desigualdad. Cabe recordar que la medición de Zacatecas se basó en gasto y no en ingreso corriente, como el obtenido de los cuadros del Coneval. La razón del gasto es parecida en ambos casos, en Zacatecas rural los no pobres y no vulnerables tienen un gasto 4.2 veces superior a los pobres extremos, a nivel nacional es 4.4 veces. La polarización social identifica al porcentaje de pobla-

$154 \frac{\text { PRIMER SEMESTRE } 2015}{\text { MIGRACIÓN Y DESARROLLO NÚM. } 24}$ 
ción según el grado de marginación social calculado por Conapo. Son municipios polarizados si menos de 20 por ciento de su población reside en localidades de grado «medio» de marginación y cada extremo («alto» y «muy alto»; «bajo» $\mathrm{y}$ «muy bajo») cuenta con más de 30 por ciento. Los municipios con polo de alta marginación son los que tienen más de 70 por ciento de su población en localidades de «alta» $\mathrm{y}$ «muy alta» marginación. Los de polo de baja marginación tienen más de 70 por ciento de su población en localidades de «baja» $y$ «muy baja» marginación. Municipios sin polo son los que no están en ninguna de las situaciones anteriores, es decir, la distribución de su población es más pareja entre los distintos estratos de marginación. El grado de polarización indicado en la columna de la Ehrurzac fue calculado con base en datos del Conapo (2011) referidos a 2010, y se refiere a la población total del estado. Vemos que la población del estado reside principalmente en municipios sin polo (59.5 por ciento), otro porcentaje importante se ubicaba en polos de baja marginación y sólo 0.3 por ciento vivía para 2010 en municipios con más de 70 por ciento de población en alta y muy alta marginación.

El índice de percepción de redes sociales es interesante al compararlo con el nacional. Una persona tendrá un grado de percepción alto si respondió que le sería fácil o muy fácil conseguir ayuda de la gente de la localidad ante diferentes opciones que se le presentaron, como conseguir dinero prestado, que alguien la cuide en caso de enfermedad, que le presten dinero para cubrir gastos médicos mayores, etcétera. En el medio rural de Zacatecas, 21.4 por ciento de los entrevistados mostró un alto grado de percepción, muy superior al nivel nacional. Aquellos con grado medio de percepción son quienes tuvieron igual número de respuestas positivas (fácil o muy fácil) que negativas (difícil o muy difícil) sobre la posibilidad de obtener ayuda ante los diferentes escenarios que se le plantearon. Su grado de percepción será bajo si considera que le sería difícil o imposible conseguir ayuda en la mayoría de las situaciones. La mayoría de la población rural tuvo un grado bajo de percepción de redes sociales. La gran diferencia se muestra con el dato nacional en percepción media de redes, lo que quizá tenga que ver con una modificación en las opciones de respuestas consideradas en la Ehrurzac. En más de 50 por ciento de los hogares se eliminó la opción de respuesta «ni fácil ni 
difícil», considerada en la ENIGH, pues se observó que a la gente le costaba trabajo entender qué significaba eso. Además, como el cuestionario es extenso, era pesado ofrecer tantas opciones de respuesta para cada reactivo considerado en esta sección. Era cansado tanto para el encuestador como para el entrevistado.

\section{CUADRO 4}

Indicadores de contexto territorial (Grado de cohesión social)

\begin{tabular}{|c|c|c|}
\hline INDICADORES & $\begin{array}{l}\text { CONEVAL } \\
\text { NACIONAL } \\
2010\end{array}$ & $\begin{array}{c}\text { EHRURZAC } \\
2009\end{array}$ \\
\hline Coeficiente de Gini & 0.51 & 0.33 \\
\hline $\begin{array}{l}\text { Razón de gasto entre la población pobre extrema y la población no } \\
\text { pobre y no vulnerable }\end{array}$ & 4.4 & 4.2 \\
\hline \multicolumn{3}{|l|}{ Grado de polarización social* } \\
\hline Población en municipios polarizados & 9.5 & 0.7 \\
\hline Población en municipios con polo de alta marginación & 4.3 & 0.3 \\
\hline Población municipios con polo de baja marginación & 59.5 & 39.5 \\
\hline Población en municipios sin polo & 26.8 & 59.5 \\
\hline \multicolumn{3}{|l|}{ Índice de percepción de redes sociales } \\
\hline Población con alto grado de percepción de redes sociales & 9.9 & 21.4 \\
\hline Población con grado medio de percepción de redes sociales & 68.8 & 1.9 \\
\hline Población con grado bajo de percepción de redes sociales & 21.3 & 76.7 \\
\hline
\end{tabular}

*A nivel nacional se refiere a «población en entidades...». El grado de polarización para Zacatecas se obtuvo a partir de los indicadores de marginación por localidad calculados por el Consejo Nacional de Población para 2010 .

El contexto territorial desde luego que es importante para explicar la pobreza, pero lo es más en el sentido de los recursos naturales locales. Como vemos, en Zacatecas la polarización social es baja; no obstante, la pobreza es alta. La desigualdad medida a través de Gini es baja; en nuestro caso, indicaría que entonces la mayoría es igualmente pobre. A veces la concentración de recursos productivos genera cierto dinamismo en las localidades, fuentes de empleo, hay trabajo todo el año para el resto de la gente.

$156 \frac{\text { PRIMER SEMESTRE } 2015}{\text { MIGRACIÓN Y DESARROLLO NÚM. } 24}$ 
El cuadro 5 muestra la contribución de cada uno de los indicadores de carencia social en la explicación de la pobreza. También se presentan las carencias que más afectan al grupo de los no pobres, pues según la definición del Coneval, una persona puede ser no pobre a pesar de padecer carencias sociales. Entre los pobres, la carencia que más contribuye a esa situación es el acceso a la seguridad social (25.6 por ciento); le sigue el acceso a la alimentación (24.1 por ciento). Entre los pobres extremos, la carencia de mayor contribución es el acceso a la alimentación (23.4 por ciento), seguida del acceso a la seguridad social (22.2 por ciento), la carencia de servicios básicos en la vivienda también tiene una participación significativa (19.5 por ciento). Para los no pobres, sólo estarían los vulnerables por carencias sociales: casi 40 por ciento de su situación de vulnerabilidad se explica por la falta de seguridad social; seguiría el rezago educativo, que aporta 15.7 por ciento, el de menor contribución promedio es la carencia de alimentos.

\section{CUADRO 5}

Localidades rurales de Zacatecas: contribución de cada indicador de carencia social, 2009 (\%)

\begin{tabular}{|c|c|c|c|c|c|c|c|}
\hline GRUPO & $\begin{array}{l}\text { REZAGO } \\
\text { EDUCATIVO }\end{array}$ & $\begin{array}{l}\text { ACCESO } \\
\text { A LOS } \\
\text { SERVICIOS } \\
\text { DE SALUD }\end{array}$ & $\begin{array}{l}\text { ACCESO } \\
\text { ALA } \\
\text { SEGURIDAD } \\
\text { SOCIAL }\end{array}$ & $\begin{array}{l}\text { CALIDAD Y } \\
\text { ESPACIOS } \\
\text { DE LA } \\
\text { VIVIENDA }\end{array}$ & $\begin{array}{c}\text { SERVICIOS } \\
\text { BÁSICOS } \\
\text { EN LA } \\
\text { VIVIENDA }\end{array}$ & $\begin{array}{c}\text { ACCESO A LA } \\
\text { ALIMENTACIÓN }\end{array}$ & TOTAL \\
\hline Pobres & 9.9 & 9.0 & 25.6 & 14.7 & 16.7 & 24.1 & 100.0 \\
\hline Pobres extremos & 9.3 & 8.9 & 22.2 & 16.8 & 19.5 & 23.4 & 100.0 \\
\hline No pobres & 15.7 & 13.8 & 37.6 & 12.1 & 12.2 & 8.5 & 100.0 \\
\hline
\end{tabular}

Fuente: Estimaciones con base en la Ehrurzac.

Los datos del cuadro 5 permiten enfocar las medidas de política pública prioritarias para superar la pobreza. El acceso a seguridad social proviene de trabajos estables, se tiene que pensar en la forma de beneficiar con esto, a través de algún fondo de aportación solidaria, a los jornaleros agrícolas y trabajadores por cuenta propia (muchos de ellos pequeños productores agropecuarios). Garantizar el acceso a la alimentación no equivale a regalar alimentos 
o despensas, sino también a fomentar la producción de huevo, leche y derivados, carne, frutas, verduras, granos básicos, plantas y frutos silvestres, como tuna y nopal en Zacatecas. Se debe invertir en la conservación y ampliación no sólo de éstos, sino en general de los recursos naturales con que cuenta el estado. Hay que invertir en mejorar la infraestructura de riego pero también en la captación de agua de lluvia. Se debe invertir en servicios al medio ambiente a través de programas de empleo, para que los propios habitantes rurales reforesten sus regiones. Toda medida de este tipo, enfocada hacia los recursos, tendrá efectos multiplicadores que no sólo beneficiarían a los habitantes rurales, sino también a los no rurales. Urge en México el apoyo a la producción de alimentos para recuperar la soberanía alimentaria. Urge ver a la gente del campo como actores clave para el desarrollo. Un desarrollo alternativo al actual, que privilegia y favorece a la gran empresa.

El acceso a servicios de salud entre la población rural se debe principalmente al Seguro Popular, lo cual está bien, pero no es suficiente. Éste debe aumentar la calidad en el servicio y la cobertura de enfermedades. El rezago educativo también ha mejorado a través del programa Oportunidades y campañas de alfabetización de adultos (muchas madres de familia de becarios de este programa han concluido su primaria o secundaria en el sistema abierto; aunque sea cuestionable la calidad de la educación que reciben, obtienen el grado y eso impacta en el indicador). Sin duda, es fundamental invertir en educación. Se debe garantizar a todo pueblo la posibilidad de contar con un educador, hay localidades con muy pocos niños, adonde no mandan maestro. El Estado tiene que cumplir con esta obligación, especialmente en aquellas localidades aisladas, en las que es prácticamente imposible que los niños y niñas se trasladen a otra localidad.

\section{Migración, pobreza y Remesas}

Zacatecas es considerada como una de las entidades más atrasadas del país por su nivel de desarrollo económico. De forma resumida, su economía descansa en la ganadería extensiva, los granos básicos y la migración de mano

$158 \frac{\text { PRIMER SEMESTRE } 2015}{\text { MIGRACIÓN Y DESARROLLO NÚM. } 24}$ 
de obra hacia otras entidades del país y, sobre todo, a Estados Unidos. El turismo también es importante, aunque se concentra en la ciudad capital.

El atraso económico incide sobre la capacidad para retener a su población: hasta 2005 era una de las entidades con menor tasa de crecimiento demográfico, aunque para 2010 se frena esa tendencia. Para este año sólo seis de sus municipios perdieron población, mientras que en 2000, 34 habían tenido tasas de crecimiento negativas y en 2005 el número había aumentado a 41 (Padilla, 2012).

En la entidad, la migración internacional se ha constituido, después de una larga trayectoria histórica, en un motor importante para la economía estatal. En 2007 recibió 687.1 millones de dólares por concepto de remesas internacionales, equivalentes a 11.43 por ciento del PIB local, la proporción más alta en el país (Moctezuma, 2011: 166). Para 2010, fue la entidad con el mayor índice de intensidad migratoria a Estados Unidos, en ese año 11.04 por ciento de sus viviendas recibieron remesas de aquel país (Conapo, 2012). La mayor parte de las personas que emigran a Estados Unidos en la entidad provienen de localidades rurales: entre 2005 y 2010, del total de personas que salieron a Estados Unidos, 58 por ciento tenían ese tipo de residencia; entre 2000 y 2005 ese porcentaje fue de 63.8, esto según los datos de las muestras del Conteo y Censo de Población de 2005 y 2010.

En este apartado vinculamos la recepción de remesas de los hogares con su situación de pobreza. Hallazgos al respecto indican que si bien las remesas son una fuente importante de ingreso para las familias perceptoras, su impacto en la reducción de la pobreza es limitado debido a que constituyen un fondo salarial, y como tal, contribuyen a mejorar el nivel de vida de los hogares perceptores y sólo de manera reducida generan movilidad social (Canales, 2008).

Según resultados de la Ehrurzac, 29.4 por ciento de los hogares rurales de Zacatecas reciben remesas, ${ }^{5}$ cifra mayor a la de la muestra del Censo de Población de 2010 para el sector rural (17.1 por ciento). En los hogares percep-

${ }_{5}^{5}$ Este indicador incluye las transferencias monetarias que recibieron los hogares por parte de hijos e hijas, o cónyuges, del jefe(a) de familia, que radicaban en Estados Unidos, más el dinero por concepto de pensiones. Si se agrega el número de hogares que recibieron trans- 
tores de remesas residía, para 2009, 26.5 por ciento de la población total captada en la muestra. En la gráfica 3 se comparan los datos de pobreza rural de Zacatecas (con remesas, sin remesas y el promedio rural estatal) con la situación de los hogares rurales a nivel nacional.

En Zacatecas, los hogares rurales perceptores de remesas son menos pobres que los no perceptores. En los primeros, 50.6 por ciento de la población eran pobres multidimensionales, en los segundos, 64.7 por ciento. Además, la incidencia de pobreza en estos hogares también es menor al promedio estatal (61 por ciento) y nacional (64.8 por ciento). Como se aprecia en la gráfica 3 , los niveles de pobreza moderada son semejantes en cada uno de los grupos destacados, por tanto, es la pobreza extrema la que marca diferencia. Ésta afecta sólo a 11.5 por ciento de la población con remesas, comparado con 24.7 por ciento de los no perceptores, 21.2 por ciento del promedio rural estatal y 23.9 por ciento del promedio rural nacional. La situación de pobreza de estos últimos es parecida al promedio estatal y nacional.

La población no pobre puede ser vulnerable por carencias sociales, por ingreso, o bien ni pobre ni vulnerable, tal como se ha indicado anteriormente. Encontramos que la vulnerabilidad por carencias sociales es especialmente aguda en los hogares perceptores de remesas, 48 por ciento de esta población estaba en tal condición, comparado con 31 por ciento en los hogares sin remesas, 35.5 por ciento del promedio estatal y 28.8 por ciento del promedio nacional. En el cuadro 6 se muestra cuáles son las carencias que afectan a estos hogares.

Las carencias sociales que afectan más a la población de hogares con remesas, en comparación con el resto, son el rezago educativo (42 por ciento) y el acceso a servicios de salud (35.6 por ciento). El primero se explica por la mayor proporción de adultos mayores en estos hogares: 33.8 por ciento de su población tiene 50 años o más, en cambio, sólo 16.4 por ciento de los no perceptores se ubicaron en ese rango para 2009. En el trabajo de campo se constató que la gente de mayor edad en el medio rural tiene bajos niveles

ferencias esporádicas de otros parientes o amigos que vivían en aquel país, el porcentaje de hogares receptores aumenta a 33.5 por ciento.

$160 \frac{\text { PRIMER SEMESTRE } 2015}{\text { MIGRACIÓN Y DESARROLLO NÚM. } 24}$ 
educativos porque antes no había escuelas o bien sólo se podía cursar hasta 3ero o 4to grado de primaria, y el umbral para superar el rezago educativo en este grupo de edad es, según la metodología del Coneval, primaria terminada. El menor acceso relativo que tienen a servicios de salud puede vincularse con la mayor capacidad para pagar emergencias médicas, gracias a las remesas, de ahí un posible menor interés por darse de alta en las instituciones públicas.

\section{GRÁFICA 3}

Distribución de la población según situación de pobreza (porcentaje)*

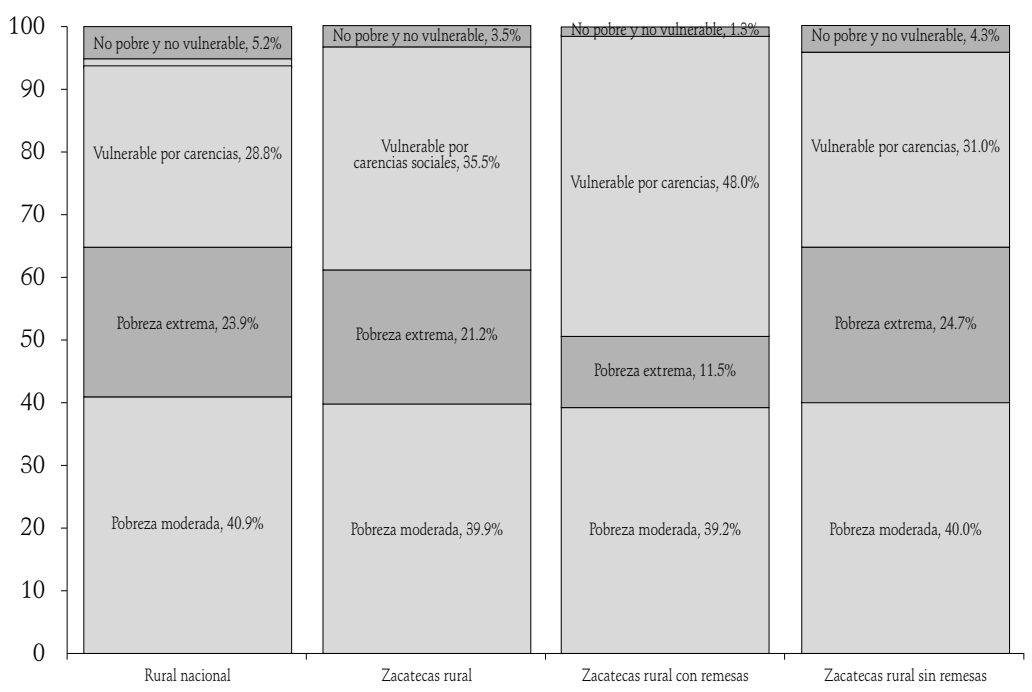

*El dato rural nacional se refiere a 2010, los de Zacatecas a 2009.

Fuente: Coneval (2011) y Encuesta a Hogares Rurales de Zacatecas, 2009.

En el cuadro 6 se puede apreciar, además, que los hogares perceptores están en mejor situación con respecto a los otros grupos en cuanto a calidad y servicios básicos en la vivienda; y tienen menor carencia en acceso a la alimentación que los hogares sin remesas. Sus niveles de ingreso (gasto en el caso de Zacatecas) también son mayores, pues sólo 12.7 por ciento de su población tenía un ingreso menor a la línea de bienestar mínimo y 50.6 


\section{CUADRO 6}

Pobreza multidimensional: indicadores de privación social

\begin{tabular}{|c|c|c|c|c|}
\hline \multirow[b]{2}{*}{ INDICADORES (PORCENTAJE DE PERSONAS) } & \multirow{2}{*}{ 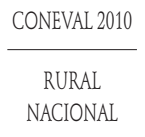 } & \multicolumn{3}{|c|}{ EHRURZAC 2009} \\
\hline & & $\begin{array}{c}\text { RURAL } \\
\text { ZACATECAS }\end{array}$ & $\begin{array}{l}\text { RURALZAC } \\
\text { CON REMESAS }\end{array}$ & $\begin{array}{l}\text { RURAL ZAC SIN } \\
\text { REMESAS }\end{array}$ \\
\hline \multicolumn{5}{|l|}{ Privación social } \\
\hline Población con al menos una carencia & 93.6 & 96.5 & 98.7 & 95.7 \\
\hline $\begin{array}{l}\text { Población con al menos tres carencias so- } \\
\text { ciales }\end{array}$ & 50.3 & 62.0 & 54.1 & 64.9 \\
\hline Indicadores de carencia social & & & & \\
\hline Rezago educativo & 33.9 & 33.9 & 42.0 & 30.9 \\
\hline Carencia por acceso a servicios de salud & 32.2 & 30.5 & 35.6 & 28.7 \\
\hline Carencia por acceso a la seguridad social & 81.9 & 85.3 & 84.2 & 85.7 \\
\hline $\begin{array}{l}\text { Carencia por calidad y espacios de la vi- } \\
\text { vienda }\end{array}$ & 29.2 & 41.7 & 26.1 & 47.3 \\
\hline $\begin{array}{l}\text { Carencia por acceso a los servicios básicos } \\
\text { en la vivienda }\end{array}$ & 46.6 & 46.2 & 39.6 & 48.5 \\
\hline $\begin{array}{l}\text { Carencia por acceso a la alimentación } \\
\text { Bienestar }\end{array}$ & 33.6 & 59.6 & 52.2 & 62.2 \\
\hline $\begin{array}{l}\text { Población con un ingreso inferior a la lí- } \\
\text { nea de bienestar mínimo }\end{array}$ & 35 & 23.1 & 12.7 & 26.8 \\
\hline $\begin{array}{l}\text { Población con un ingreso inferior a la línea } \\
\text { de bienestar }\end{array}$ & 66 & 61.0 & 50.6 & 64.7 \\
\hline
\end{tabular}

Fuente: Coneval (2011) y Encuesta a Hogares Rurales de Zacatecas, 2009.

por ciento menor a la de bienestar (comparados con 26.8 por ciento y 64.7 por ciento en los hogares sin remesas). Obviamente, el impacto mayor de las remesas se ubica en este rubro.

La mirada multidimensional de la pobreza permite sopesar mejor los efectos de las remesas internacionales en la superación de ésta. Los indicadores de carencia social se relacionan con bienes y servicios que el Estado debe proveer a sus ciudadanos. Vemos que las remesas son importantes para mejorar la condición de pobreza de los hogares vía mayor ingreso y mejoras en la vivienda. No obstante, el que parte importante de la población perceptora de estos recursos pertenezca a grupos de mayor edad, aminora su impacto, ya que laboran en actividades por cuenta propia (sin acceso a seguridad social) y tienen bajos niveles educativos porque en su tiempo no había escuela.

$162 \frac{\text { PRIMER SEMESTRE } 2015}{\text { MIGRACIÓN Y DESARROLLO NÚM. } 24}$ 


\section{CONCLUSIONES}

Sin duda la pobreza rural se explica en gran medida por la profunda crisis que padece el campo desde hace por lo menos 40 años. En la década de los cincuenta la preocupación de académicos e instituciones era promover la industrialización en países en vías de desarrollo, ello se hizo en detrimento del desarrollo agropecuario, a través de sistemas de intercambio desiguales, con precios relativos favorables al sector industrial, lo que generó la transferencia de excedente de un sector a otro. Se generó una situación de desequilibrio favorable al crecimiento de actividades no agrícolas, concentradas en las ciudades, generando un proceso acelerado de urbanización. La llamada revolución verde, estrategia puesta en marcha en la década de los cincuenta, que promovió el uso de fertilizantes químicos, semillas mejoradas y maquinización de la producción para aumentar la productividad, tuvo efectos diferenciados sobre los productores rurales. Los que tenían capacidad para adaptarse al cambio tecnológico y acceso a irrigación se vieron favorecidos, los pequeños productores salieron perdiendo. La década de los sesenta marca el inicio de la crisis del agro que se padece actualmente, y que se ha profundizado por el desmantelamiento del Estado y la apertura comercial vigentes en México desde finales de la década de los ochenta.

Los costos de haber ignorado el equilibrio que debe procurarse entre desarrollo agrícola e industrial se están viviendo actualmente: una pobreza testaruda que no cede a las transferencias monetarias focalizadas hacia los pobres.

Esa crisis ha llevado al país a la pérdida de soberanía alimentaria, pero aun así se insiste en seguir ignorando al campo. La gente que sigue en localidades rurales se ha vuelto cada vez más dependiente de ingresos ajenos a las actividades agropecuarias, como las transferencias, el comercio y el trabajo asalariado fuera de la parcela.

El enfoque multidimensional propuesto por el Coneval y retomado en este trabajo para dar cuenta de la pobreza en las zonas rurales del estado tiene la virtud de asumir que ésta se asocia con diferentes dimensiones de la vida de las personas. Los recursos monetarios son importantes para cubrir 
las necesidades básicas, pero no es suficiente concentrarse en ellos para determinar la magnitud del problema. Para superar la pobreza, el Estado debe asumir su responsabilidad de proveer los bienes y servicios reconocidos como derechos constitucionales.

\section{Bibliografía}

Aragonés, Ana María (2004), «Migración y explotación de la fuerza de trabajo en los años noventa: saldos del neoliberalismo», en Blanca Rubio (coord.), El sector agropecuario mexicano frente al nuevo milenio, México, UnAm y Plaza y Valdés.

Boltvinik, Julio (2003), "Conceptos y métodos para el estudio de la pobreza», Comercio Exterior, vol. 53, núm. 5.

Canales, Alejandro (2008), Vivir del norte. Remesas, desarrollo y pobreza en México, México, Consejo Nacional de Población.

Consejo Nacional de Población (Conapo) (2011), Índices de marginación por entidad federativa y municipio 2010, en http://www.conapo.gob.mx/es/CONAPO/Indices_de_Marginacion $(12 / 12 / 2012)$.

(2012), Índices de intensidad migratoria México-Estados Unidos 2010, México, Conapo, en http://www.omi.gob.mx/es/OMI/Indices_de_intensidad_migratoria_Mexico-Estados_Unidos_2010 (05/03/2014).

Consejo Nacional para la Evaluación de la Política de Desarrollo Social (Coneval) (2010), Metodología para la medición multidimensional de la pobreza en México, México, Coneval.

(2011), «Resultados de pobreza a nivel nacional y por entidad federativa, 2008-2010», en http://www.coneval.gob.mx/medicion/Paginas/Medici\%C3\% B3n/Pobreza-2010.aspx (consultado el 12 de enero de 2013).

GonZÁlez Montes, Soledad (1994), «Mujeres, trabajo y pobreza en el campo mexicano: una revisión crítica de la bibliografía reciente», en Javier Alatorre (coord.), Las mujeres en la pobreza, México, Grupo Interdisciplinario sobre Mujer, Trabajo y Pobreza y Colegio de México.

Gordon, Dave (2004), "Poverty, Death and Disease», en Paddy Hillyard, Christina Pantazis, Steve Tombs y Dave Gordon (eds.), Beyond Criminology: Taking Harm Seriously, Londres, Pluto.

$164 \frac{\text { PRIMER SEMESTRE } 2015}{\text { MIGRACIÓN Y DESARROLLO NÚM. } 24}$ 
Hopenhayan, Martin (2003), La pobreza en conceptos, realidades y políticas: una perspectiva regional con énfasis en minorías étnicas, Santiago, Chile, CEPAL, en http://www. iidh.ed.cr/comunidades/diversidades/docs/div_enlinea/Pobreza\%20afros.pdf (consultado el 25 de junio de 2013).

KAY, Cristóbal (2006), «Rural Poverty and Development Strategies in Latin America», Journal of Agrarian Change, vol. 6, núm. 4.

KLeIn, Emilio (2012), "Condicionantes laborales de la pobreza rural en América Latina», en Fernando Soto Baquero y Emilio Klein (coords.), Políticas de mercado de trabajo y pobreza rural en América Latina, Santiago, FAO.

Mathus, Marco Aurelio (2008), "Principales aportaciones teóricas sobre la pobreza», en http://www.eumed.net/rev/cccss/02/mamr.htm (25/06/2013).

Moctezuma, Miguel (2011), La transnacionalidad de los sujetos: dimensiones, metodologías y prácticas convergentes de los migrantes en Estados Unidos, México, Miguel Ángel Porrúa, UAZ.

PADILLA, Juan Manuel (2012), "La demografía de Zacatecas al inicio del siglo XXI», en Rodolfo García Zamora y Francisco Javier Contreras Díaz (eds.), Seminario estatal de universitarios: por una nueva estrategia de desarrollo integral para Zacatecas, México, Universidad Autónoma de Zacatecas.

Romero, Alberto (2002), Globalización y pobreza, Colombia, Ediciones Unariño, en http://www.eumed.net/cursecon/libreria/AR-glob-libro.pdf

RuBio, Blanca (2004), «La fase agroalimentaria global y su repercusión en el campo mexicano", Comercio Exterior, vol. 54, núm. 11.

Sen, Amartya (2000), Desarrollo y libertad, México, Planeta.

SPicker, Paul (2009), «Definiciones de pobreza: doce grupos de significados», en Paul Spicker y David Gordon (eds.), Pobreza: un glosario internacional, Buenos Aires, CLACSO.

Tetreault, Darcy (2012), "Alternativas para salir de la pobreza en el México rural», en Humberto Márquez, Roberto Soto y Edgar Záyago (coords.), Visiones del desarrollo, México, Miguel Ángel Porrúa y Universidad Autónoma de Zacatecas. 
